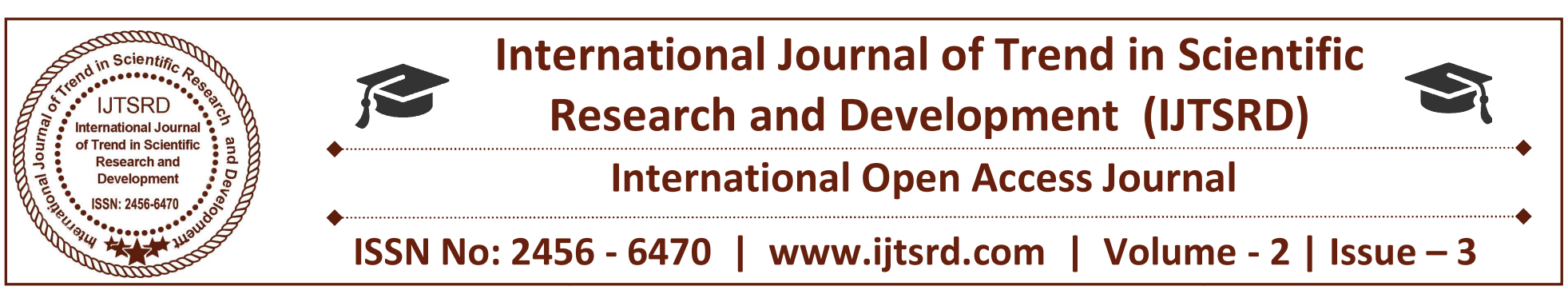

\title{
De-Identification Technology Involves Web Mining
}

\author{
G. Sneka \\ B.E (IV-CSE), Ponnaiyah \\ Ramajayam College of \\ Engineering and Technology, \\ Thanjavur, Tamil Nadu, India
}

\author{
H. Sharmila \\ B.E (IV-CSE), Ponnaiyah \\ Ramajayam College of \\ Engineering and Technology, \\ Thanjavur, Tamil Nadu, India
}

\author{
R. Banumathi \\ Assistant Professor, Ponnaiyah \\ Ramajayam College of \\ Engineering and Technology, \\ Thanjavur, Tamil Nadu, India
}

\section{ABSTRACT}

Many data owners are required to release variety of data in real world application it has vital importance of discovery valuable information stay behind the data. However existing we focus on the deidentification policy which is the common privacy preserving approach. By using de-identification policy a continues balance between privacy protection and data utility can be achieved by choosing the appropriate. We propose one parallel algorithm "SKY FILTER POLICY GENERATOR" that can be filtered the optimized data" web ranking algorithm" provide the user preferred data. Each user has their own privacy username and password, through the sky filter (data analytic operator) user willing data given to the user. Each user is identified by de identification policy.

Keywords: sky filter policy generator, web ranking algorithm, data analytic

\section{INTRODUCTION}

Many data owners are required to release the data in a variety of real world application, since it is of vital importance to discovery valuable information stay behind the data. However, existing re-identification attacks on the AOL and ADULTS datasets have shown that publish such data directly may cause tremendous threads to the individual privacy. Thus, it is urgent to resolve all kinds of re-identification risks by recommending effective de-identification policies to guarantee both privacy and utility of the data. Deidentification policies is one of the models that can be used to achieve such requirements, however, the number of de-identification policies is exponentially large due to the broad domain of quasi-identifier attributes. To better control the tradeoff between data utility and data privacy, skyline computation can be used to select such policies, but it is yet challenging for efficient skyline processing over large number of policies. We propose one parallel algorithm called SKY-FILTER-MR, which is based on Map Reduce to overcome this challenge by computing skyline largescale de-identification policies, is represented by bitstring. To provide sufficient background knowledge for our work, we discuss research efforts in privacy preserving data publication, risk and utility cost, skyline queries with a special focus on parallel processing, and discovery of deidentification policies. To use the De-Identification technology for optimized willing data given to the user through the sky filter policy generator. To better control the tradeoff between data utility and data privacy, skyline computation can be used to select such policies, but it is yet challenging for efficient skyline processing over large number of policies. We propose one parallel algorithm called" SKY-FILTER POLICY GENERATOR". The optimal balance between data privacy and data utility has been paid more and more attention. To meet the multilevel needs of users for privacy and data utility, the discovery of deidentification policies is of paramount importance. To provide sufficient background knowledge for our work, we discuss research efforts in privacy preserving data publication, risk and utility cost, skyline queries with a special focus on parallel processing, and discovery of deidentification policies. 


\section{DESIGN}

Web Services

Adapter module prepared in creating an example Stateless adapter module. The Web Services module will have the following properties: package: com.acompany.wespa Web Services module will use the interfaces defined for the example Stateless .my sc type Interface used by the client, acting on the Web Services module: My Service Capability Interface used by the Web Services module, acting on the client: My Service Capability Listener Method implemented by the Web Services module for application-initiated requests, defined in the My Service Capability interface: my Method Req enable Network Triggered Events disable Network Triggered Events Methods invoked by the Web Services module for application-initiated requests, defined in the My Service Capability Listener interface, and implemented by the client: my Method Res my Method End deactivate Interface used by the Web Services module, acting on the client: My Service Capability Network Triggered Event Listener Methods invoked by the Web Services module for network-triggered requests, defined in the My Service Capability Network Triggered Event Listener interface, and implemented by the client :my Deliver Network Triggered Event Method deactivate

Access Centre

Overview

of database

systems

including database design, Entity Relationship data modeling, the relational model of data and SQL, as well as an overview of some database products.

View

The views module allows adminis-trators and site designers to create, manage, and display lists of content. Each list managed by the views module is known as a "view", and the output of a view is known as a "display". Displays are provided in either block or page form, and a single view.

Multi end client

The purpose of this module is to provide the user interface and view functions for the system. This is the software with which the user directly interacts. It communicates with the server to retrieve and modify persistent data when necessary.

Policy Generator

Policy Generator assists administrators in describing role-based policies with browsing resource information and user information, and it stores the descriptions in the form of XACML in Policy Repository.

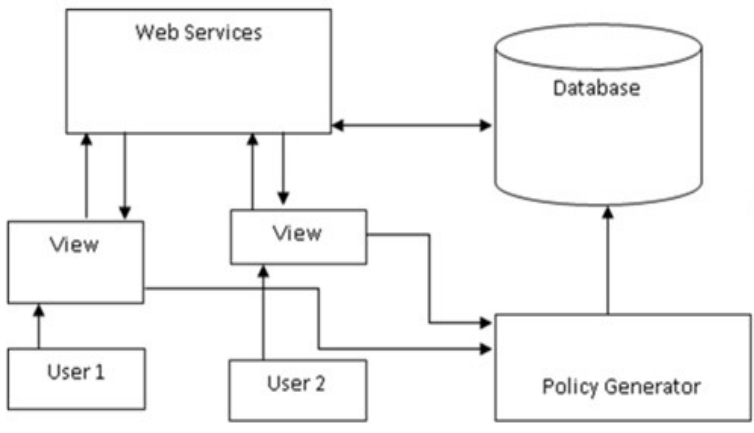

IMPLEMENTATION

Here we proposed the system de- identification technology involves web mining the information are being connected with person identity which are give the user willing optimized data through the" SKY FILTER POLICY GENERATOR" through the algorithm called the "web ranking algorithm" can analyze the how much time the user can view the data and what kind of data they view.

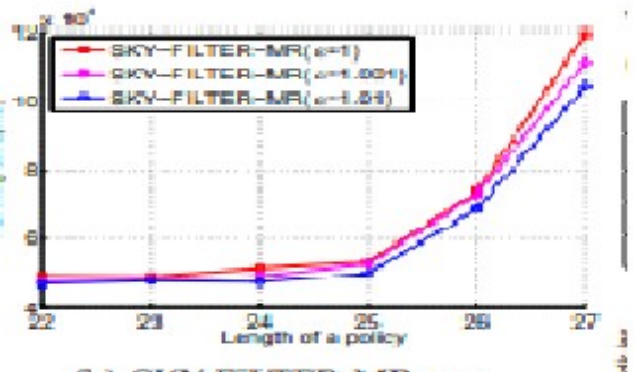

(b) SKY-FILTER-MR vS.E

Figure: Graph for sky filter

Policy generator can filter the user willing optimized data given to the user

\section{RELATED WORK}

APPLET: a privacy-preserving framework for location-aware recommender system. (Xindi MA1,2, Hui LI2, Jianfeng MA1,2, Qi JIANG2, Sheng GAO3, Ning XI2* \& Di LU1 OCT -2016) Location-aware recommender systems that use location-based ratings to produce recommendations have recently experienced a rapid development and draw significant attention from the research community. However, current work mainly focused on high-quality recommendations while underestimating privacy issues, which can lead to problems of privacy. Such problems are more prominent when service providers, who have limited computational and storage resources, leverage on cloud platforms to fit in with 
the tremendous number of service requirements and users. In this paper, we propose a novel framework, namely APPLET, for protecting user privacy information, including locations and recommendation results, within a cloud environment. Through this framework, all historical ratings are stored and calculated in ciphertext, allowing us to securely compute the similarities of venues through Paillier encryption, and predict the recommendation results based on Paillier, commutative, and comparable encryption. We also theoretically prove that user information is private and will not be leaked during a recommendation.

Efficient Discovery of De-identification Policies Through a Risk-Utility Frontier.( BradelyMalin, Jiuyong Li, Raymonds Heatherly, Weiyi Xia, Xiaofeng Ding) Modern information technologies enable organizations to capture large quantities of person-specific data while providing routine services. Many organizations hope, or are legally required, to share such data for secondary purposes (e.g., validation of research findings) in a de-identified manner. In previous work, it was shown deidentification policy alternatives could be modeled on a lattice, which could be searched for policies that met a pre specified risk threshold (e.g., likelihood of reidentification). However, the search was limited in several ways. First, its definition of utility was syntactic - based on the level of the lattice - and not semantic - based on the actual changes induced in the resulting data. Second, the threshold may not be known in advance. The goal of this work is to build the optimal set of policies that trade-off between privacy risk $(\mathrm{R})$ and utility $(\mathrm{U})$, which we refer to as a $\mathrm{R}-\mathrm{U}$ frontier. To model this problem, we introduce a semantic definition of utility, based on information theory, that is compatible with the lattice representation of policies. To solve the problem, we initially build a set of policies that define a frontier. We then use a probability guided heuristic to search the lattice for policies likely to update the frontier. To demonstrate the effectiveness of our approach, we perform an empirical analysis with the Adult dataset of the UCI Machine Learning Repository. We show that our approach can construct a frontier closer to optimal than competitive approaches by searching a smaller number of policies. In addition, we show that a frequently followed de-identification policy (i.e., the Safe Harbor standard of the HIPAA Privacy Rule) is suboptimal in comparison to the frontier discovered by our approach.
A Simple and Practical Algorithm for Differentially Private Data Release (Moritz Hardt, Katrina Ligett,Frank Mcsherry) We present a new algorithm for differentially private data release, based on a simple combination of the Exponential Mechanism with the Multiplicative Weights update rule. Our MWEM algorithm achieves what are the best known and nearly optimal theoretical guarantees, while at the same time being simple to implement and experimentally more accurate on actual data sets than existing techniques. Fault tolerance, locality optimization and load balancing. Second, a large variety of problems are easily expressible as Map Reduce computations. For example, Map Reduce is used for the generation of data for Google's production web search service, for sorting, for data mining, for machine learning, and many other systems. Third, we have developed an implementation of Map Reduce that scales to large clusters of machines comprising thousands of machines. Sensitive statistical data on individuals are ubiquitous, and publishable analysis of such private data is an important objective. When releasing statistics or synthetic data based on sensitive data sets, one must balance the inherent tradeoff between the usefulness of the released information and the privacy of the affected individuals. Against this backdrop, differential privacy $[4,11,5]$ has emerged as a compelling privacy definition that allows one to understand this tradeoff via formal, provable guarantees. In recent years, the theoretical literature on differential privacy has provided a large repertoire of techniques for achieving the definition in a variety of settings . However, data analysts have found.

PrivBayes: Private Data Release via Bayesian Networks Cecilia M. Procopiuc, Divesh Srivastava, Jun Zhang,Graham Cormode, Xiaoki ) Privacypreserving data publishing is an important problem that has been the focus of extensive study. The stateof-the-art goal for this problem is differential privacy, which offers a strong degree of privacy protection without making restrictive assumptions about the adversary. Existing techniques using differential privacy, however, cannot effectively handle the publication of high-dimensional data. In particular, when the input dataset contains a large number of attributes, existing methods require injecting a prohibitive amount of noise compared to the signal in the data, which renders the published data next to useless. To address the deficiency of the existing methods, this paper presents PRIVBAYES, a differentially private method for releasing high- 
dimensional data. Given a dataset D, PRIVBAYES first constructs a Bayesian network $\mathrm{N}$, which (i) provides a succinct model of the correlations among the attributes in D and (ii) allows us to approximate the distribution of data in $\mathrm{D}$ using a set $\mathrm{P}$ of low dimensional marginal's of D. After that, PRIVBAYES injects noise into each marginal in $\mathrm{P}$ to ensure differential privacy, and then uses the noisy marginal's and the Bayesian network to construct an approximation of the data distribution in D. Finally, PRIVBAYES samples tulles from the approximate distribution to construct a synthetic dataset, and then releases the synthetic data. Intuitively, PRIVBAYES circumvents the curse of dimensionality, as it injects noise into the low-dimensional marginal's in $\mathrm{P}$ instead of the high dimensional dataset D. Private construction of Bayesian networks turns out to be significantly challenging, and we introduce a novel approach that uses a surrogate function for mutual information to build the model more accurately. We experimentally evaluate PRIVBAYES on real data, and demonstrate that it sig

A Data-and Workload-Aware Algorithm for Range Queries Under Differential Privacy.(Chao LilC, Gerome Miklau, Michael Hay, Yue Wang) We describe a new algorithm for answering a given set of range queries under -differential privacy which often achieves substantially lower error than competing methods. Our algorithm satisfies differential privacy by adding noise that is adapted to the input data and to the given query set. We first privately learn a partitioning of the domain into buckets that suit the input data well. Then we privately estimate counts for each bucket, doing so in a manner well-suited for the given query set. Since the performance of the algorithm depends on the input database, we evaluate it on a wide range of real datasets, showing that we can achieve the benefits of data-dependence on both "easy" and "hard" databases. Differential privacy [8, 9] has received growing attention in the research community because it offers both an intuitively appealing and mathematically precise guarantee of privacy. In this paper we study batch (or noninteractive) query answering of range queries under differential privacy. The batch of queries, which we call the workload, is given as input and the goal of research in this area is to devise differentially private mechanisms that offer the lowest error.

\section{CONCLUSION}

We study the recommendation on a great number of de-identification policies using Map Reduce. Firstly, we put forward an effective way of policy generation on the basis of newly proposed definition, which can decreases the time of generating policies and the size of alternative policy set dramatically. Secondly, we propose SKY-FILTER-POLICY GENERATOR, which is are analyze the user willing optimized data through the database we propose algorithm called web ranking algorithm used to analyze the user preferred data, to answer skyline de-identification policies efficiently.

\section{FUTURE ENHANCEMENT}

To further improve the performance, a novel approximate skyline computation scheme was proposed to prune unqualified policies using the approximately domination relationship with approximate skyline, the power of filtering in the policy space generation stage was greatly strengthened to effectively decrease the cost of skyline computation over alternative policies. Extensive experiments over both real life and synthetic datasets demonstrate that our proposed SKY-FILTER-POLICY GENERATOR algorithm substantially outperforms the baseline approach by faster in the optimal case, which indicates good scalability over large policy sets.

\section{REFERENCES}

1) B. C. M. Fung, K. Wang, R. Chen, and P. S. Yu, "Privacy-preserving data publishing: A survey of recent developments," ACM Comput. Surv., vol. 42, no. 4, pp. 14:1-14:53, 2010.

2) $=$ K. Benitez, G. Loukides, and B. Malin, "Beyond safe harbor: Automatic discovery of health information de-identification policy alternatives," in IHI, 2010, pp. 163-172.

3) K. E. Emam, "Heuristics for de-identifying health data," IEEE Security and Privacy, vol. 6, no. 4, pp. 58-61, 2008.

4) L. Sweeney, "k-anonymity: A model for protecting privacy," International Journal of Uncertainty, Fuzziness and Knowledge-Based Systems, vol. 10, no. 05, pp. 555-570, 2002. d K. Shim, "Approximate

5) W. Xia, R. Heatherly, X. Ding, J. Li, and B. Malin, "Efficient discovery of de-identification policies through a risk-utility frontier," in CODASPY, 2013, pp. 59-70.

6) X. MA, H. Li, J. Ma, Q. Jiang, S. Gao, N. Xi, and D. Lu, "Applet: A privacy-preserving framework for location-aware recommender system," Sci China Inf Sci, vol. 59, no. 2, pp. 1-15, 2016. 